\title{
Coronavirus and dentistry - fighting the pandemic
}

\author{
Akanksha Raj, Neetha J. Shetty, Sweta Pradhan \\ Manipal College of Dental Sciences, Mangalore, Manipal Academy of Higher Education, India
}

\begin{abstract}
The coronavirus disease (COVID-19) has challenged health professions and systems, and developed different speeds of reaction and types of response around the world. Coronavirus is an enveloped virus with positive-sense single-stranded RNA. Coronavirus infection in humans mainly affects the upper respiratory tract, and to a lesser extent, the gastrointestinal tract. Clinical symptoms of coronavirus infections can range from relatively mild (similar to the common cold) to severe (bronchitis, pneumonia, and renal involvement). The role of dental professionals in preventing the transmission of COVID-19 is critically important. While all routine dental care has been suspended in countries experiencing COVID-19, the need for organized urgent care delivered by teams provided with appropriate personal protective equipment has become a priority. Dental professionals can also contribute to medical care. Major and rapid reorganization of both clinical and support services is not simple. Dental professionals considered a moral duty to reduce routine care due to fear of spreading COVID-19 among their patients, but were understandably concerned about financial consequences. Amidst the explosion of information available online and through social media, it is difficult to identify reliable research evidence and guidance; therefore, moral decisions must be made.
\end{abstract}

HIV AIDS Rev 2020; 19, 4: 217-221 DOI: https://doi.org/10.5114/hivar.2020.101493

Key words: COVID-19, SARS CoV-2, dentistry.

\section{Introduction}

As of December 12, 2019, a highly infectious pneumonia started to spread in Wuhan, China. At the beginning of January 2020, officials declared COVID-19 or novel corona virus as the causative pathogen for the disease $[1,2]$. The World Health Organization has called this novel viral pneumonia as coronavirus disease (COVID-19). The International Committee for the Taxonomy of Viruses (ICTV) also named this new coronavirus as SARS CoV-2. It soon became one of the toughest public health challenges in the modern world, spreading throughout the globe to more than 200 countries. On January 30,2020 , the WHO declared the outbreak of COVID-19 as an international public health emergency [1-3].

Clinical symptoms of COVID-19 may vary from case to case, but the most common symptoms attributed to this disease are fever, continuous dry cough, fatigue, and myalgia. In more severe cases, computed tomography (CT) scans of abdomen and chest reported findings, such as consolidative pulmonary opacity and peripheral ground glass appearances. Also, less common clinical symptoms, which are associated with COVID-19 include headache, excess sputum production, hemoptysis, and diarrhea [3, 4].

In 1968, Almeida et al. [5] first described a newly discovered single-stranded RNA virus, with a diameter of $120 \mathrm{~nm}$. Because of its appearance under an electron microscope, scientists decided to name this new group of viruses as "corona

Article history:

Received: 04.08.2020

Received in revised form: 24.08 .2020

Accepted: 26.08.2020

Available online: 30.11 .2020
International Journal of HIV-Related Problems

HIV \& AIDS

$R$ e $v$ i e w 


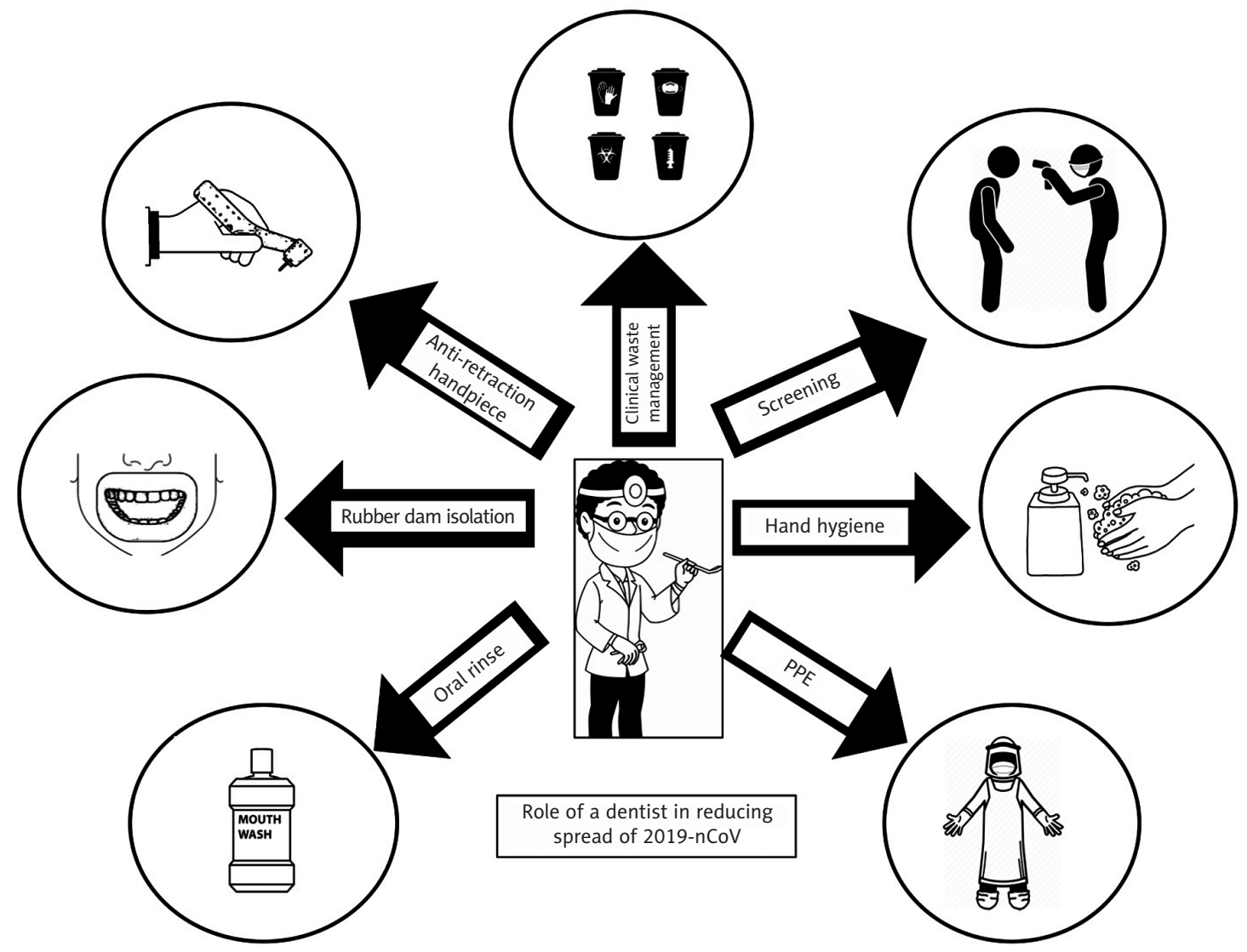

Figure 1. Role of dentists in reducing spread of COVID-19 disease

virus", owing to its fringe of projections on the outer surface, which resembled solar corona [6].

Coronaviruses are prone to recombination and mutations; therefore, approximately 40 different variants of coronaviruses have been found, which mainly infect humans, other mammals, and birds. There have been evidences suggesting that the COVID-19 pathogen firstly originated from bats species and got transferred to intermediate hosts, such as wild dogs, pangolins, and snakes. Human spread has been attributed to contaminated meat products from traditional wildlife markets in Wuhan [6-9].

\section{Potential routes of transmission and its spread}

Possible routes of transmission involve coughing and sneezing, with virus droplets and microdroplets from infected individuals. It can also spread through oral and nasal contact, and via ocular mucosal membranes [7]. Airborne route can become a major route of transmission, since it leads to formation of aerosols, which may contain virus particles, as clinical studies indicate that most of dental procedures involving use of rotary handpieces generate considerable amount of contaminated and potentially infectious droplets and aerosols [7-10]. One study suggested its possible feco-oral route, in which real-time PCR test of nasopharyngeal/oropharyngeal swabs and samples from stool collected after one week of illness, detected RNA of the virus and tested positive for COVID-19. However, more research and studies are needed to confirm the potential feco-oral route of transmission [10,11].

Direct passage and spread of novel coronaviruses through respiratory droplets and fomites are quite common. Its incubation period varies from 7 to 24 days and some cases present with no clinical symptoms. Therefore, a bilateral risk of being exposed to virus transmitted through the oral cavity and respiratory tract during a dental visit increases [12].

Frequent contamination with saliva, blood, and other body fluids as well as the use of sharp and high-speed rotary instruments, clearly multiplies the risk of infection in a dental set up [11-13]. Generated aerosols can settle on surfaces of instruments and dental equipment, further increasing the risk of cross-contamination between dentists and patients. Hence, a highly effective and robust cross infection control protocols become the need of the hour [11-13].

\section{Clinical manifestations}

Primary non-specific reported symptoms of 2019-nCoV infection at the prodromal phase are malaise, fever, and dry cough. The most commonly reported signs and symptoms include fever (98\%), cough (76\%), dyspnea (55\%), and myalgia or fatigue $(44 \%)$. Unlike patients with other human corona- 


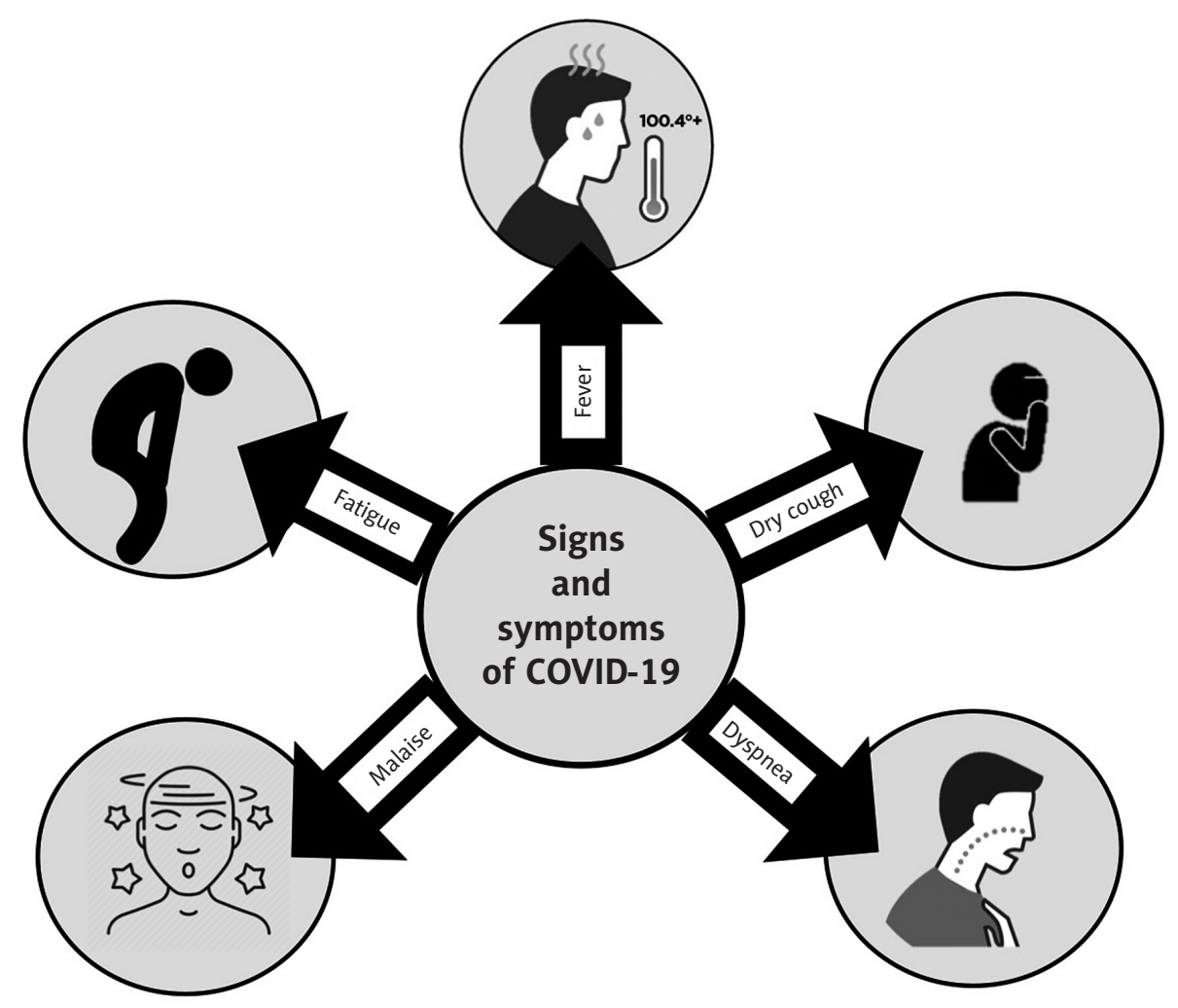

Figure 2. Signs and symptoms of COVID-19 virus

virus infections (e.g., SARS-CoV), upper respiratory tract and intestinal manifestations, such as sore throat, rhinorrhea, and diarrhea in 2019-nCoV infection patients are infrequent.

\section{Role of dentists and patients in reduc- ing the spread of COVID-19 [14-17]}

\section{Screening of patients}

Strict protocols should be followed for patients' screening, so that identification of potential high-risk COVID-19 patients become feasible, and prevention measures can be adopted immediately. Also, routine dental treatment at an early stage of infection must be discouraged, and patients must be urged for quarantine or self-isolation. Following, there are some of the screening measures, which need to be incorporated before attending any patient in an operatory:

- body temperature measurement of each patient with contact-free infrared forehead thermometer;

- request every patient to complete a questionnaire to determine if the patient had any symptoms of COVID-19 in the past 2 weeks, such as fever, persistent cough or sneezing, and difficulty in breathing. History regarding social and public gathering need to be additionally noted;

- any contact with a COVID-positive patient should be recorded.

\section{Hand hygiene}

Regular hand hygiene by washing hands with soap and water is one of the most frequent and emphasized measure that has been suggested by the WHO to limit the spread of coronavirus. Dental professionals must avoid touching their own eyes, nose, and mouth, until it is safe to do so.

An alcohol-based hand rubs with at least $60 \%$ isopropanol or ethanol has also been documented as a simple and effective cross infection control technique, which can aid in inactivating enveloped viruses, including coronaviruses.

\section{Personal protective measures for dental professionals}

To ensure safety of both dental healthcare professionals and patients, it becomes extremely important to incorporate effective PPE procedures in dental care centers. It is highly recommended to use barrier protections, such as full-length fluid repellant gowns, FFP3 respirators, full face shield or visor, and gloves, especially for high- risk patients.

\section{Before starting any dental procedure}

Aerosol generation is practically inevitable during most of dental procedures. Thus, through various preventive mea- 
sures, such as pre-operative use of antiseptic mouth rinses, it is important to reduce the viral load in aerosols and droplets. Povidone iodine solution possess strong bactericidal and viricidal properties, and has shown $99.99 \%$ activity against pathogens, such as influenza, Ebola, MERS, and coronavirus SARS causing oral and respiratory tract infections. The available evidence provides a strong support for the safe use of povidone iodine oral solution as a protective oropharyngeal hygiene measure for people at high-risk of oral and respiratory pathogens acquisition.

\section{Isolation using rubber dams}

Application of a rubber dam is one of the simplest and most practical ways to reduce the contamination from the oral cavity and achieve effective moisture control. The isolation of rubber dam has been shown to be effective in minimizing the outflow of saliva- and blood-contaminated aerosols, particularly during procedures using highspeed handpieces and ultrasonic instruments.

\section{Use of anti-retraction handpieces}

Using an anti-retraction handpiece results in a significant reduction in the backflow of bacteria and viruses from the oral cavity into the handpiece and dental unit tubes, as compared to a handpiece with no anti-retraction function. During dental operations, ordinary handpieces may aspire and expel the infected fluids containing bacteria and viruses, and may contaminate the air and water tubes inside the dental unit increasing the chances of cross-infection. Therefore, during the COVID-19 pandemic, the use of anti-retraction handpieces is recommended and encouraged.

\section{Strict disinfecting protocol for dental clinics}

It is vital that medical and dental teams follow an effective and strict protocol of disinfection for both operatory and community areas. As per guidelines and instructions, all surfaces in clinical areas must be washed and disinfected to the highest standards. The community areas and public facilities must be regularly cleaned and disinfected, including complete disinfection of all door handles, touch screens, monitors, and furniture, like desks and chairs. Installing improved air ventilation systems in healthcare facilities will also help to ease the elimination of airborne contaminants from clinical environment and reduce the risk of infection.

\section{Clinical waste management}

Biomedical or clinical waste should be stored in a secure provisional holding area, and must be pre-treated, washed, sterilized, and stored properly in accordance with the guidelines for all reusable instruments and materials. The medical hazardous waste after treatment of COVID-19-positive patients must be considered as clinically infectious waste and stored within a designated area in clinical waste bags. The surface of these bags should be appropriate labeled, and handled in accordance with the guidelines and requirement for the management of medical wastes.

\section{COVID-19 testing}

Various approaches for COVID-19 testing are available, and the decision to conduct a test on a suspected individual should be taken based on clinical symptoms and epidemiological variables. COVID-19 detection kits should be used in order to test high-risk and suspicious patients. Real-time PCR and medical imaging are among other tests, which can be used for a detection of COVID-19-positive patients [18-20].

\section{Treatment}

In two recently published papers, possible therapies with pharmacological treatments for COVID-19 disease have been thoroughly and systematically reviewed. Potential therapeutic interventions for COVID-19 include general supportive treatments, such as vitamins $\mathrm{A}, \mathrm{B}, \mathrm{C}, \mathrm{D}$, and $\mathrm{E}$, omega-3 polyunsaturated fatty acids, selenium, zinc, and iron. Moreover, monoclonal antibodies can also support neutralizing SARS-CoV-2 in a cell culture. Chloroquine, hydroxy-chloroquine, and promazine are the drugs that may play a promising role in the arrest of the disease [20].

\section{Conclusions}

Many changes in infection control procedures and associated dental armamentaria can be expected to arise in the post-COVID-19 world of dentistry. The extent and severity of change will be determined by an evidence and research of the best and safest practices. Prior to mandating change that will involve an extreme financial and architectural change of the current dental office, research should be conducted evaluating current available practices, methodology, and instrumentation, which can mitigate or prevent the risk of transmission, while being financially and practically expeditious. In conclusion, the COVID-19 pandemic has several immediate complications for dentistry, of which some might have further long-term impacts on clinical practice, dental education, and dental research.

\section{Conflict of interest}

The authors declare no conflict of interest with respect to the research, authorship, and/or publication of this article.

\section{References}

1. Coronavirus disease 2019 (COVID-2019) situation report - 32. World Health Organization. February 21, 2020. Available at: https:// www.who.int/docs/default-source/coronaviruse/situation-reports/ 20200221-sitrep-32-covid-19.pdf.

2. Zhu N, Zhang D, Wang W, et al. A novel coronavirus from patients with pneumonia in China, 2019. N Engl J Med 2020; 382: 727-733. 
3. Su S, Wong G, Shi W, et al. Epidemiology, genetic recombination, and pathogenesis of coronaviruses. Trends Microbiol 2016; 24 490-502.

4. Cui J, Li F, Shi ZL. Origin and evolution of pathogenic coronaviruses. Nat Rev Microbiol 2019; 17: 181-192.

5. McIntosh K. Coronaviruses: a comparative review. In: Current Topics in Microbiology and Immunology/Ergebnisse der Mikrobiologie und Immunitätsforschung. Springer: Berlin/Heidelberg 1974; pp. 85-129.

6. Peiris JSM, Yuen KY, Osterhaus ADME, Stohr K. The severe acute respiratory syndrome. N Engl J Med 2003; 349: 2431-2441.

7. Bai Y, Yao L, Wei T, et al. Presumed asymptomatic carrier transmission of COVID-19. JAMA 2020; 323: 1406-1407.

8. Van Doremalen N, Bushmaker T, Morris DH, et al. Aerosol and surface stability of SARS-CoV-2 as compared with SARS-CoV-1. N Engl J Med 2020; 382: 1564-1567.

9. Verity R, Okell LC, Dorigatti I, et al. Estimates of the severity of coronavirus disease 2019: a model-based analysis. Lancet Infect Dis 2020. doi: 10.1016/S1473-3099(20)30243-7.

10. Nejatidanesh F, Khosravi Z, Goroohi H, Badrian H, Savabi O. Risk of contamination of different areas of dentist's face during dental practices. Int J Prev Med 2013; 4: 611-615.

11. Williams GH, Pollok NL, Shay DE, Barr CE. Laminar air purge of microorganisms in dental aerosols: prophylactic procedures with the ultrasonic scaler. J Dent Res 1970; 49: 1498-1504.

12. Harrel SK, Molinari J. Aerosol and splatter in dentistry: a brief review of the literature and infection control implications. J Am Dent Assoc 2004; 135: 429-437.

13. Miller RL. Characteristics of blood-containing aerosols generated by common powered dental instruments. Am Ind Hyg Assoc J 1995; 56 670-676.

14. Guidance on preparing workplaces for COVID-19. US Department of Labor. Occupational Safety and Health Administration 2020 doi: https://www.osha.gov/Publications/OSHA3990.pdf.

15. Davidson BL. Doctor: How to reduce your vulnerability to coronavirus - when sleeping. CNN. Updated March 13, 2020. doi: https:// www.cnn.com/2020/03/12/opinions/coronavirus-vulnerabilitywhile-sleeping-bruce-davidson/index.html

16. Drosten C, Günther S, Preiser W, et al. Identification of a novel coronavirus in patients with severe acute respiratory syndrome. N Engl J Med 2003; 348: 1967-1976.

17. Gasmi A, Noor S, Tippairote T, Dadar M, Menzel A, Bjørklund G. Individual risk management strategy and potential therapeutic options for the COVID-19 pandemic. Clin Immunol 2020, 108409

18. Sanders JM, Monogue ML, Jodlowski TZ, Cutrell JB. Pharmacologic treatments for coronavirus disease 2019 (COVID-19): a review. JAMA 2020; 323: 1824-1836.

19. Testing for COVID-19 | CDC. Available online: https://www.cdc. gov/coronavirus/2019-ncov/symptoms- testing/testing.html

20. McKee DL, Sternberg A, Stange U, Laufer S, Naujokat C. Candidate drugs against SARS-CoV-2 and COVID-19. Pharmacol Res 2020 157: 104859 